\title{
La lettre volée par le diable. Lecture d'une faute de langue dans la dédicace des Fleurs du mal
}

\section{Loïc Windels}

\section{(2) OpenEdition \\ Journals}

Édition électronique

URL : http://journals.openedition.org/lcc/182

DOI : $10.4000 /$ lcc. 182

ISSN : 2430-4247

Éditeur

Université Aix-Marseille (AMU)

Référence électronique

Loïc Windels, «La lettre volée par le diable. Lecture d'une faute de langue dans la dédicace des Fleurs du mal », Les chantiers de la création [En ligne], 2 | 2009, mis en ligne le 01 avril 2015, consulté le 08 avril 2020. URL : http://journals.openedition.org/lcc/182 ; DOI : https://doi.org/10.4000/lcc.182 


\section{La lettre volée par le diable}

\section{Lecture d'une faute de langue dans la dédicace des Fleurs du mal}

Loïc WINDELS, Université Paris 8 Vincennes - Saint-Denis

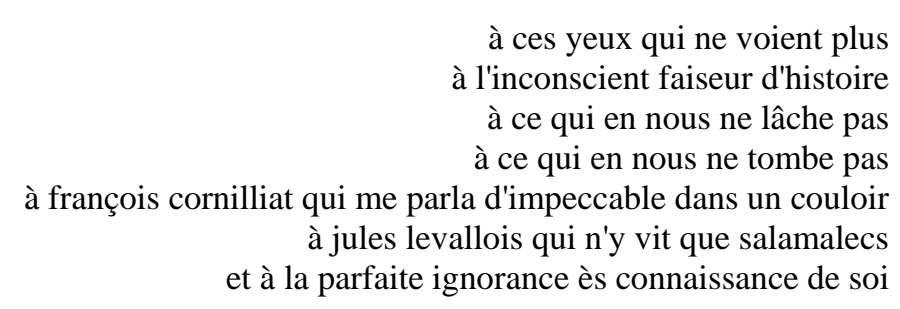

Un lecteur qui, en 1857, ouvrait un exemplaire des Fleurs du mal commençait par lire la dédicace suivante :

AU POÈTE IMPECCABLE

AU PARFAIT MAGICIEN ÈS LANGUE FRANÇAISE

À MON TRÈS-CHER ET TRÈS-VÉNÉRÉ

MAÎTRE ET AMI

THÉOPHILE GAUTIER

AVEC LES SENTIMENTS

DE LA PLUS PROFONDE HUMILITÉ JE DÉDIE

CES FLEURS MALADIVES

C. B.

Ceux qui savent m'auront deviné. Pour les autres, ils auront peut-être, et à l'instar de Baudelaire, lu une faute sans la voir. Contraction de « en les », la préposition « ès » doit, en langue française, être suivie du pluriel. Cette erreur linguistique frappant la «langue française » au cœur même d'une perfection langagière affirmée avec force (le magicien y est dit «parfait »), ce vrai lapsus linguce qui sera corrigé dès la seconde édition, mais qui, dans la première, semblait comme un clinquant monté en épingle par le hasard, le diable, ou un désir inconscient, il y a longtemps que j'avais envie, pour reprendre les termes de 
cette journée, d'en examiner la fécondité interprétative. Il y avait là un fait de langue imputable, sinon à la volonté, du moins à la plume de Baudelaire ; la perfection de son imperfection m'étonnait et je me demandai ce qu'il en adviendrait si je poussais plus avant les procédés exégétiques dont j'avais l'habitude, comme s'il se fût agi d'un trait de style ou d'un sonnet. La pensée ne s'embarrasse pas des éventuels scrupules de l'herméneute, et la facilité avec laquelle les idées venaient, se précisaient, se renforçaient me réjouit avant de me déconcerter. Quelle valeur, en effet, demeure à l'exégèse de traits de style si l'exégèse d'un coup du sort est en tout point analogue ? On amasse des indices, on les rapproche, ils se combinent. Et ? Quel garant ? La ferme décision de ne pas tout à fait raconter n'importe quoi ? Cela fait peu et je m'apprêtais déjà à prendre le ciel à partie, oui, j'allais pour m'exclamer avec Baudelaire traduisant Poe : de pareilles choses sont-elles l'opération d'un hasard indiscipliné ? Quand le hasard, bonhomme, sembla ne pas vouloir être en reste et m'offrit des passages corroborant ceux que la mémoire m'avait d'elle même proposés. Je fis une seconde pause en songeant qu'il faudrait tout de même finir par donner forme lisible à ce beau sac de liens tissés entre la faute et l'œuvre, et que le beau sac tienne en quelques pages. Farouche partisan de l'intentionnalité baudelairienne, j'avais tout de même vaguement entendu parler d'inconscient freudien... Je pourrais donc, ainsi muni d'autorité, saupoudrer mon propos de références et pouvais pour l'heure laisser la pensée se rouler dans l'interprétation. Je fus un peu effrayé de m'apercevoir que j'en venais bientôt à lire et à interpréter non plus seulement l'acte manqué, mais le hasard même, que la psychanalyse, pas plus qu'un coup de dé, n'a jamais pu bien abolir. Lecteur, m'aurais-tu suivi dans ces usines sans garde-fou ? Conscient de la difficulté, je résolus de faire, au moins pour cette fois, machine arrière, de régler le pas de ces courses à l'exégèse, et d'en livrer ici, résumés et corsetés, les résultats. Les voici donc, sans toutes les preuves, mais assagis in extremis en un plan : nous commencerons par décrire la faute en son contexte dédicatoire, puis donnerons une interprétation de cette erreur de plume comme trahison de soi par des désirs antagonistes, lecture que nous affinerons ensuite en confrontant le lapsus aux notions baudelairiennes de chute et d'ironie, avant, finalement, d'en pister les devenirs dans l'œuvre et conclure. Nous aurons même une problématique, double, et il sera souvent question d'autorité et de sincérité.

\section{Description d'une erreur en son contexte : sincérité et perfection}


On aurait envie, avant tout, de se mettre d'accord sur un point : qu'il y a bien quelque chose qui échappe à la conscience dans cette faute. Stricto sensu, c'est évident : Baudelaire n'a pas dû être ravi de se prendre a posteriori en flagrant délit de faute de langue. Mais Freud fait souvent appel, pour le convaincre qu'il y a des actes où l'inconscient est en jeu, à l'expérience de son lecteur : ces actes auraient comme une saveur différente. Et c'est en particulier une expérience commune à quiconque ouvre La Psychopathologie de la vie quotidienne que de nourrir sa lecture de ses propres exemples, qu'on les puise en soi ou en ceux qui - c'est ainsi qu'alors on se le formule - ont agité comme un chiffon ou comme un fouet leur inconscient devant nos yeux. Mais dès que nous cessons d'être un témoin, pour devenir le légataire de traces qui ne nous étaient pas toutes destinées, il ne peut plus s’agir que de soupçons. Voici les miens.

\section{1 Psychopathologie d'une erreur de relecture}

Tout d'abord, si la dédicace est célèbre et sa perfection célébrée, non moins célèbre est la méticulosité légendaire du Baudelaire correcteur d'épreuves. Et s'il se rendit coupable de rares fautes, notamment dans les publications en revue, nulle à ma connaissance ne parvint jusqu'au recueil ${ }^{1}$. Or, la dédicace fut l'objet d'un soin particulier et de surcharges particulièrement importantes ${ }^{2}$. Donc, le premier soupçon est que Baudelaire a relu cent fois sa faute sans la voir ${ }^{3}$. Le second soupçon, c'est qu'à la fin de ces relectures, en envoyant son bon à tirer, Baudelaire, au bas de la dernière épreuve, ajoute à l'intention de son éditeur : «mes yeux n'y voient plus; relisez après que toutes vos corrections seront vérifiées » (Pichois \& Dupont 971). Ses yeux n'y voyaient plus, mais encore assez pour qu'affleure un soupçon de "cécité » ${ }^{4}$. On me dira : rien que de très naturel après avoir examiné, comme le commissaire de La lettre volée qu'il avait traduite, «les planches au microscope » (Poe \& Baudelaire 100). Mais comme lui sans rien trouver : il eût fallu la lucidité d'un Dupin pour voir l'évidence de ces deux S volés par l'inconscient ou quelque

\footnotetext{
${ }^{1}$ J'excepte les fautes d'orthographe ou de conjugaison qui subsistent, mais qui peuvent être corrigées sans remaniement du vers (Bo Liu, Les "Tableaux parisiens" de Baudelaire (Paris : L'Harmattan, 2003) p.188-91, et Henk Nuiten, Les Variantes des «Fleurs du mal » et des «Épaves » de Charles Baudelaire (1821-1867). Étude de stylistique génétique (Amsterdam : Holland University Press, 1979), p. 3-37). Dans le cas du lapsus au contraire, quand les S apparaissent, la « langue » doit disparaître.

${ }^{2}$ Qu'on feuillette, pour s'en convaincre, l'édition diplomatique des Fleurs du mal (Pichois \& Dupont 966-72). Voir aussi Henk Nuiten (p.9-10), Jean Pommier, Autour de l'édition originale des Fleurs du mal (Genève : Slatkine reprints, 1968), p.114-7, et la Correspondance, notamment cette lettre adressée à son éditeur pour lui « recommande[r s]a dédicace avec un amour infini » $(C P l, I, 385)$.

${ }^{3}$ « Et comment se fait-il encore que, malgré trois corrections d'épreuves, ces erreurs m'aient échappé, comme si j'avais été frappé de cécité ? » (Freud, 2001, 274).

${ }^{4}$ Voir note précédente.
} 
Druckfehlerteufel $^{5}$. Car il y a un dernier soupçon. Ses yeux se sont bien posés sur la faute, ou plutôt, juste à côté. Non seulement il revient plusieurs fois sur cette expression dont il est visiblement satisfait lorsqu'il évoque dans une lettre «le magicien » pour se référer à Gautier ( $C P l, 1,380)$, mais son attention tourne autour de son erreur au point d'exprimer, au dos de la même épreuve, ces doutes :

pourquoi ès-science?

de science,

pourquoi ès-langue ? nous ne dirions pas : de-langue, en-langue n'écririons

(Pichois \& Dupont 972)

Cette fois, la faute, portant sur la locution figée, non sur celle forgée par Baudelaire, n'a plus d'excuses. Mais Baudelaire raye « ès-science », comme si la faute, alors trop visible, avait inconsciemment sauté à ces yeux qui ne voient plus. D'autant que Baudelaire procède ensuite à ce que Freud appellerait peut-être la réduction de la préposition complexe ${ }^{6}$. Mais elle est imparfaite et reste condensée en ce que l'article pluriel « les », qui permettrait de lever la faute, est refoulé : « ès-langue [...] en-langue ». Et entre les deux Baudelaire barre l'oralité au profit d'un amuïssement dans l'écrit. Or, s'il avait « dit » la préposition, il aurait précisément fait ce que nous faisons chaque fois que nous relisons la dédicace de 1857 : entendre ce qui manque à cette " langue » laissée au singulier. Car après « ès », il en faut un. Mais le plus important, du point de vue d'un soupçon de signature inconsciente, c'est que ce doute et ce passage du « ès » au « en » ne portent pas sur le pluriel. Pour employer, cette fois sans métaphore, un terme à consonance freudienne, l'attention est déplacée vers le tiret seul, entraînant à sa suite l'attention du relecteur ${ }^{7}$. Il y a donc bien soupçon d'acte manqué, qu'il s'agirait dès lors d'interpréter : ruse du diable ou coup de l'inconscient. Mais avant d'interpréter, il faut encore décrire l'erreur en son contexte.

\section{2 Une dédicace parfaite(ment sincère ?)}

Sous le vernis de perfection dont on gratifie généralement la dédicace, on voulut parfois s'étonner du choix de Gautier. Le problème de la sincérité fut soulevé, et dès lors, ce fut,

\footnotetext{
${ }^{5}$ Terme allemand qui signifie littéralement : « démon des fautes d'impression ».

${ }^{6} \mathrm{La}$ « réduction », procédé par lequel Freud analyse les mots d'esprit, « vise à annuler rétroactivement le processus de condensation » à l'origine du mot d'esprit comme du rêve (Freud, 1995, 76).

7 Freud décrit par exemple ainsi le "déplacement du rêve ": "pendant le travail du rêve, l'intensité psychique passe des pensées et représentations auxquelles elle convient légitimement à d'autres pensées et représentations qui, à mon sens, ne peuvent prétendre à une telle mise en valeur " (Freud, 1991, 84). Déplacement qu'il voit également à l'œuvre dans le lapsus et dans le mot d'esprit.
} 
comme chaque fois qu'il l'est, bien difficile de le reléguer au dossier des questions réglées. Le soupçon d'ironie est une sorte d'hydre : sitôt un doute tranché, deux repoussent. Je ne puis ici détailler. Mais si l'on se souvient que la dédicace fut « discutée, convenue et consentie avec le magicien" $(C P l, I, 380)$ et que Baudelaire le pria, sur un riche exemplaire qu'il lui destinait en privé, de croire à son "amitié et [à son] admiration véritables » $(O C, I, 830)$, on a envie de dire, avec Michel Butor, que si réserves il y a, elles ne font pas le poids face à «cette "sincérité" douloureusement, laborieusement conquise, cet aveu public par lequel on s'engage » (Butor 21). Et il me semble beaucoup plus intéressant, et fructueux, de considérer qu'il y a bien là souci de sincérité, plutôt qu'hypothétique manque. Gageons donc que Baudelaire, dans cette dédicace, a désiré être sincère. Arguant, dès lors, que «les erreurs grammaticales [...] souillent les meilleures intentions » $(O C, I I, 328)$, une première interprétation serait de considérer que Baudelaire se trahit dans une faute opérant le retour des réserves refoulées ${ }^{8}$. Mais si c'est pour découvrir que rares sont les admirations sans réserve aucune, on aurait envie d'ajouter : et après ? Laissons donc pour le moment le problème d'une ironie possible, nous $\mathrm{y}$ reviendrons. Retenons seulement qu'il y a sincère désir de sincérité, et que celui-ci se double, plus évident encore, d'un parfait souci de perfection qui prétend donner, en sa forme même, une description de Gautier comme « un des maîtres les plus sûrs en matière de langue et de style » (152).

\section{3 Perfection langagière contre facilité de plume}

Figurez-vous, je vous prie, la langue française à l'état de langue morte. [...] Dans quels auteurs supposez-vous que les professeurs, les linguistes d'alors, puiseront la connaissance des principes et des grâces de la langue française ? Sera-ce, je vous prie, dans les capharnaüms du sentiment ou de ce que vous appelez le sentiment ? Mais ces productions, qui sont vos préférées, seront, grâce à leur incorrection, les moins intelligibles [...] car il n'y a rien qui soit plus obscur que l'erreur et le désordre. Si dans ces époques [futures] les poésies de Théophile Gautier sont retrouvées par quelque savant amoureux de beauté, je devine, je comprends, je vois sa joie. Voilà donc la vraie langue française ! (151)

\footnotetext{
${ }^{8}$ « On est étonné de constater que le penchant à la vérité est beaucoup plus fort qu'on n'est porté à le croire. Il faut peut-être voir une conséquence de mes recherches psychanalytiques dans le fait que je suis devenu presque incapable de mentir. Toutes les fois où j'essaie de déformer un fait, je commets une erreur ou un acte manqué qui [...] révèle mon manque de sincérité » (Freud, 2001, 278).
} 
Vous venez de lire un extrait du Théophile Gautier de Baudelaire ${ }^{9}$. Et maintenant figurezvous Baudelaire se rendant compte qu'il a livré au public son premier recueil de poésie avec, trônant en première page, la langue française malmenée. Figurez-vous-le et devinez, comprenez, voyez son désarroi. Figurez-vous sa main qui saisit un crayon et tremblante, rageuse ou philosophe - vous trouverez vous-même l'adjectif qui vous semblera le mieux convenir à la personnalité de l'intéressé et à sa situation - griffonne sur quelques exemplaires les S manquants, « au prix de l'étrangeté » nous précise Claude Pichois (OC, $I$, 829). Voilà la scène qu'il nous faut garder en mémoire, celle du moment où, après la publication, les yeux de Baudelaire se dessillent. Voilà le blocage dont on voudrait trouver la cause ${ }^{10}$. Mais pour l'heure, revenons à nos professeurs linguistes.

Cette profession de foi d'un classicisme austère peut étonner sous la plume de celui qui définissait sa modernité par le romantisme. Mais elle n'étonne plus guère dès lors qu'on se rappelle que Baudelaire est loin de réclamer tout l'héritage. Choisir Gautier, c'était choisir son romantisme, celle d'un certain «magisme », certes, mais indissociable d'un souci de la langue et d'une conscience de l'écriture en opposition stricte avec l'épanchement sentimental et la facilité de plume ${ }^{11}$. Choisir Gautier, c'était se démarquer de la « canaille » élégiaque $(O C, I, 182)$, de Musset en particulier et son «torrent bourbeux de fautes de grammaire et de prosodie» $(C P l, I, 675)^{12}$. Ainsi s'explique cette insistance sur la perfection et l'impeccabilité du poète. Comme Poe, Gautier est du côté d'une poésie consciente d'elle-même et de ses effets. À l'inverse, « erreur », « désordre » « incorrection », «torrent bourbeux », « négligence »: on pourrait multiplier les occurrences qui font de la faute de langue le critère rhétorique frappant de nullité les tenants d'une poésie inspirée, d'une poésie du cœur qui ferait l'économie du «travail par lequel une rêverie devient un objet d'art » (675). On comprend donc l'ironie de ce lapsus qui fait basculer Baudelaire du mauvais côté, au moment même où il cherche à marquer la frontière qui le sépare de la «mauvaise poésie », frontière qui autrement serait peut-être

\footnotetext{
${ }^{9}$ Plus exactement d'une notice de 1861, plus courte que le Théophile Gautier de 1859 , qui semblait quant à lui avoir déjà pris acte de la faute, puisqu'il se terminait, en capitales d'imprimerie, par la description de Gautier en « PARFAIT HOMME DE LETTRES », allusion possible à la future version de la dédicace.

${ }^{10}$ Pour un exemple de cécité de relecture qui se prolonge jusqu'à ce qu'une nouvelle situation, écartant le danger qui était la cause du refoulement, rende la vue à l'intéressé et débloque l'interprétation, voir Sigmund Freud, Psychopathologie de la vie quotidienne (Paris : Payot \& Rivages, 2001), p.134-6.

${ }^{11}$ Le terme de «magisme » apparait dans cette Fusée : « culte (magisme, sorcellerie évocatoire) [...] De la langue et de l'écriture, prises comme opérations magiques, sorcellerie évocatoire » (OC, $I, 658)$.

${ }^{12}$ On trouve dans la Correspondance de Flaubert la même opposition entre Gautier et Musset, au profit du premier, et à peu près pour les mêmes raisons.
} 
bien difficile à délimiter nettement ${ }^{13}$. Certes, Baudelaire corrigera la faute en 1861 et la ligne incriminée sera désormais dédiée « au parfait magicien ès lettres françaises ». Mais ce sera, cette fois, au prix d'une imprécision, imprécision coupable de ne pas tenir ensemble « l'idée et l'expression » $(O C, I I, 152)$ et qu'il ne s'est sans doute jamais tout à fait pardonnée $^{14}$.

\section{Interprétation de l'erreur : autorité de l'homme bon en public}

Baudelaire avait donc trouvé la formule, mais le hasard voulut qu'elle fût fautive et nous sommes désormais en mesure d'analyser en quoi cette ironie du sort est bien, selon les termes d'une alternative baudelairienne, « hystérique » ou « satanique » $(O C, 1,286)$. Car les superlatifs de la dédicace n'y peuvent mais, et Baudelaire en 1856 semblait vaticiner quand il écrivait, à propos de ces « âmes de choix » poursuivies par le guignon :

Elles perfectionneront la prudence, boucheront toutes les issues, matelasseront les fenêtres contre les projectiles du hasard; mais le Diable entrera par une serrure ; une perfection sera le défaut de leur cuirasse, et une qualité superlative le germe de leur damnation. (OC, II, 297)

\section{1 Le « défaut de la cuirasse " : un lapsus qui tombe bien (mal)}

Qu'on me pardonne un calembour, mais ce lapsus de plume ne pouvait en effet mieux tomber, ni faire tomber de plus haut : la réussite de la faute signe l'échec de la formule ${ }^{15}$. Et c'est à présent vers Le Mot d'esprit et son rapport à l'inconscient qu'il nous faudrait nous tourner, pour en emprunter la technique de réduction à quoi fut fait allusion plus haut. D'après ce qui précède, on peut facilement décrire l'expression «magicien ès langue française » comme la condensation, d'une part, de l'idée que Baudelaire se fait de la poésie et, d'autre part, de la locution consacrée « docteur ès sciences ${ }^{16}$ ». L'idée, que l'on a trouvée

\footnotetext{
13 Thématiquement, notamment. Et, du point de vue de la réception, rappelons que Villemain pour exprimer, à la parution des Fleurs, que le romantisme a tout dit et que Baudelaire vient trop tard et bien mal, ne comparera pas, pour le dévaluer, le nouveau recueil à l'España de Gautier, mais bien aux Contes d'Espagne et d'Italie. Et ce, malgré la dédicace. Par ailleurs, il est bien évident que la distinction entre bons et mauvais poètes est de Baudelaire seul (voir, par ex. OC, II, 232).

${ }^{14}$ Voici le passage du Théophile Gautier : «Sa gloire est double et une en même temps. Pour lui l'idée et l'expression ne sont pas deux choses contradictoires qu'on ne peut accorder que par un grand effort ou par de lâches concessions. À lui seul peut-être il appartient de dire sans emphase: Il n'y a pas d'idées inexprimables!»

${ }^{15}$ «La ressemblance entre un lapsus et un jeu de mot peut aller très loin » (Freud, 2001, 100). Mais Freud parle là du phénomène inverse : non pas du mot d'esprit tombé lapsus, mais du lapsus s'avérant mot d'esprit une fois tombé des lèvres.

${ }^{16}$ Ne laissons point parler trop haut notre propre « désir de monter en grade » $(O C, I, 682)$ : Littré donne pour exemples non «docteur », mais «bachelier» ou « licencié ès lettres ». En revanche, il faut, au regard du
} 
dans les carnets intimes ${ }^{17}$, est exactement celle exprimée à propos de Gautier dans la longue notice qu'il lui consacra en 1859 : «Manier savamment une langue, c'est pratiquer une espèce de sorcellerie évocatoire » $(O C, I I, 118)$. Et l'on vient de voir qu'elle visait à démarquer Baudelaire de la «mauvaise» poésie romantique, type Musset. Mais l'idée véhiculée par l'expression " docteur ès sciences » est autre. Certes, elle vise à renforcer le paradoxe apparent d'une poésie comprise comme pratique magique et savante ${ }^{18}$. Mais elle est moins une idée qu'une valeur, empruntée à un discours d'autorité, le discours légitimant de l'université française. Gautier reçoit son diplôme de magicien, mais c'est Baudelaire qui le décerne. Et l'on comprend ici (on aurait pu le voir ailleurs) que le désir de perfection est aussi un « désir de monter en grade ${ }^{19}$ » et d'être investi d'une part de l'autorité reconnue au dédicataire. Ainsi, les « projectiles du hasard » ne sont pas perdus pour tout le monde, et il y a bien une cible à l'ironie de l'inconscient. Mais ce n'est pas Gautier qui est visé, c'est Baudelaire lui-même qui, pour filer la métaphore vers le bas, se tire une balle dans le pied.

On peut à présent proposer une interprétation plus convaincante de la faute : un désir de légitimité trahi par un désir contraire d’illégitimité. Baudelaire a souci d'asseoir son autorité, que son inconscient sape aussitôt, savamment. Et si la cécité dura jusqu'à publication, pour cesser avec elle, c'est - interprétation du blocage - que le coup porté à la légitimité du poète n'avait de sens que devant témoins : le bourreau de soi-même officie en public, afin que le public atteste de la déchéance et de l'humiliation due à cette « double postulation simultanée » des désirs antagonistes (OC, I, 682). En ce sens, la ruse de l'inconscient fut efficace. Il n'est qu'à consulter, à défaut de magicien, une référence ès langue française. Et quand on ouvre Le bon usage à la page « ès », on lit :

L'oubli de la valeur primitive amène des auteurs assez nombreux à employer Ès avec un singulier. Puisqu'il s'agit d'un archaïsme un peu prétentieux, n'est-il pas logique de l'employer de préférence à bon escient ? On n'imitera donc pas les auteurs suivants: Au parfait magicien ès langue française (BAUDEL., Fl. du m., Dédic., $1^{\text {re }}$ version), etc. (Grevisse 867)

Voilà, Monsieur Baudelaire, ce que c'est que l'autorité. On ne joue pas les Icare impunément, ou l'on connaît bien vite le «Châtiment de l'orgueil ». Ce que Baudelaire

doute évoqué, privilégier « sciences » à « lettres », même si ce dernier viendra en effet prendre la place de la «langue » en 1861.

17 Voir note 11.

${ }^{18}$ Ainsi, dans le même passage : « Il y a dans le style de Théophile Gautier une justesse [...] qui fait songer à ces miracles produits dans le jeu par une profonde science mathématique » $(O C, I I, 118)$.

${ }^{19}$ Citons plus amplement ce fragment que nous avons déjà croisé et croiserons encore : « Il y a dans tout homme, à toute heure, deux postulations simultanées, l'une vers Dieu, l'autre vers Satan[,] un désir de monter en grade [et] une joie de descendre » $(O C, 1,682-3)$. 
rejoue dans le lapsus, c'est très exactement ce qu'il décrit dans ce dernier poème : d'avoir voulu « monter trop haut », le voilà, pour peu qu'ils sachent leur grammaire, devenu « des enfants la joie et la risée » $(O C, I, 20)^{20}$. Mais que le processus inconscient de l'erreur puisse être aussi bien décrit par un poème nous remet aussitôt une puce à chaque oreille. La première s'interroge : ne faudrait-il pas, comme dans le poème et, du reste, comme à peu près dans toute l'œuvre de Baudelaire, moraliser la faute ${ }^{21}$ et percevoir dans cette chute publique l'écho d'une « joie de descendre » (682) ? La seconde lui rappelle que Baudelaire aimait à mystifier ses contemporains, qu'il propagerait en Belgique de diffamantes rumeurs sur son compte et relierait explicitement dans un poème en prose «l'esprit de mystification » aux « impulsion mystérieuse [et] intuition fortuite » qui nous échappent (285-6). Et nos puces de demander à l'unisson si cette trahison patente de Baudelaire par son inconscient ne trahirait pas à son tour une fidélité à soi plus profonde.

\section{2 Le " germe de la damnation ": le lapsus comme (in)conscience de la Chute}

Commençons $^{22}$ par nous rappeler ce sincère désir de sincérité qui présidait à la composition de la dédicace, malgré le surcroît d'autorité visé. Considérons ensuite que ce désir s'accompagne d'un souci d'exactitude : il s'agit que la parole colle à la pensée et lui donne son nom ${ }^{23}$. Maintenant observons la transformation que l'erreur et sa rectification font subir à la langue : de la formule au lapsus, c'en est fait de sa belle unité. Langue française. Langues françaises. Non seulement le sens s'égare, mais en imposant les marques du pluriel, la correction de la faute obligeait Baudelaire à tenir dans la forme ce double langage qu'il avait si soigneusement évité dans le fond. On me dira, ce n'est

\footnotetext{
${ }^{20}$ Le protagoniste du poème est « un docteur des plus grands » et il déchoit dans la folie pour avoir prétendu qu'il pouvait, après l'avoir lui aussi «poussé bien haut», rabaisser Dieu en l'attaquant «au défaut de l'armure ». L'analogie est donc doublement parfaite et m'est au passage secourable en ce qu'elle justifie $a$ posteriori le choix de « docteur » plutôt que « bachelier ».

${ }^{21}$ Une idée contraire est exprimée dans les Maximes consolantes sur l'amour (OC, 1, 549). Mais il n'est point, je pense, besoin d'aller chercher la revendication baudelairienne du droit de se contredire pour écarter cette occurrence. Car, d'une part, elle est insérée dans un contexte de misogynie badine et d'autre part, j'ai rappelé ailleurs (Windels 2008) que ce texte datait, pour Baudelaire, précisément d'une époque d'avant la conviction que l'homme porte la faute en lui de façon irrémédiable.

${ }^{22}$ J'avais initialement prévu un détour par une faute plus ancienne. En 1854 en effet, Baudelaire laissait déjà une erreur se glisser dans une dédicace, celle, publiée en revue, de traductions de Poe à la belle-mère de ce dernier, qu'il érigeait en modèle des bontés maternelles. Force m'est d'avouer que le souvenir de la Chute et la question de la bonté de l'homme apparaissent moins nettement dans la dédicace du recueil que dans cet antécédent où le « lapsus d'esprit » portait précisément sur les mots « goodness » et « godness » (sic). Pour des raisons de temps, il nous faut toutefois renoncer à cette excursion et nous en tenir à la seule erreur des Fleurs. Le lecteur devra donc, pour l'extraordinaire antécédent de 1854 se reporter à un article à paraître (Windels 2010) et pour ce qui suit, me faire un peu confiance. Le veut-il ? Allons-y.

${ }^{23}$ Voir notes 14 et 24.
} 
qu'image. Eh bien prenons l'image au sérieux et reprenons notre calembour : le lapsus fait, à la lettre, tomber la « langue » dans le multiple, ce qui, pour Baudelaire, est le signe même de la Chute ${ }^{24}$. Certes, même un novice sait que «toute erreur n’est pas une hérésie ». Mais l'homme étant tombé, et la langue avec lui, il serait bon de ne pas s'en laisser accroire. Et si, comme j’aime à le penser, le désir de sincérité n’est pas étranger à la suppression involontaire de ces maudits $S$, celle-ci peut à son tour renvoyer, par une manière d'allégorie inconsciente, à « la suppression de l'idée du péché originel ». Et cela, c'est précisément « la grande hérésie moderne » (CPl, $I, 337)$. Voici donc les deux derniers soupçons que je voudrais voir peser sur cette erreur de plume : le refus de l'ironie comme cause de la faute et la faute comme image du péché. Reste à nourrir ces soupçons d'indices tirés de la dédicace même. Or des traces de la Chute et de son déni s'y laissent deviner et se rattachent au lapsus de deux manières : par métaphore ou par contiguïté.

La première lecture voudrait donc débusquer l'esprit du mal derrière ces $\mathrm{S}$ écrits à l'encre sympathique ${ }^{25}$. Mais si je criais au diable, je craindrais qu'on ne m'écoute plus, et qu'on ne voie dans ce dæemon ex machina qu'une pure chimère d'herméneute, résidu hasardeux des fumées que l'interprétation soulève en avançant. Aussi, sur ces pluriels archaïsants à propos desquels Baudelaire expose ses doutes, sur ce S qui disparaît de l'exergue pour qu'y ressuscite le mal, ou sur cet autre S qui « danse » sur une épreuve, comme un serpent, pour s'amuïr quelques pages plus loin à la rime : silence ${ }^{26}$. Mais deux messes basses : d'une part, l'échange entre le sens et le son, et plus généralement l'abandon du lien logique au profit de l'analogie, caractérisent le fonctionnement de la pensée inconsciente. ${ }^{27}$ Le diable, d'autre part, a souvent représenté pour Baudelaire ce qui, dans le hasard, semble doté d'une intention. ${ }^{28}$ C'est ainsi qu'il lui fait tenir « les fils qui nous

\footnotetext{
${ }^{24}$ «Qu'est-ce que la chute ? Si c'est l'unité devenue dualité, c'est Dieu qui a chuté » (OC, I, 688). Sur ce point par trop elliptique, voir par exemple Jacques Salvan, « Le sens de la chute dans l'œuvre de Baudelaire » (The French Review, Déc. 1960) p.127-31 et 136-9.

${ }^{25}$ L'oubli des S valant dès lors oubli du « Lucifer latent [...] installé dans tout cœur humain » (OC, II, 168).

${ }^{26}(C P l, I, 378)$; (Dupont \& Pichois, 961, 970-95) et (OC, I, 29) pour le serpent.

${ }^{27}$ Freud va souvent plus loin : «Sous la pression de la censure, n'importe quelle sorte de lien entre deux choses devient assez bon pour assurer le remplacement par allusion, et le déplacement opéré à partir d'un élément vers n'importe quel autre est autorisé. Il existe une chose tout particulièrement frappante et caractéristique du travail du rêve, c'est le remplacement des associations internes (similitude, lien de causalité, etc.) par celles qu'on appelle externes (simultanéité, contiguïté dans l'espace, homophonie)» (Freud, 1995, 309-10).

${ }^{28}$ J'en veux pour preuve ce «projectile » promu « Diable » sitôt passé «par une serrure » (OC, II, 297). Baudelaire, qui exprima souvent le nouveau par l'obsolète, semble parfois confier au Diable l'intuition d'un inconscient. Flaubert fera de même, dans La Légende de Saint Julien. Mais dans le cas de Baudelaire cette figure permettait surtout de conserver l'hésitation anachronique entre pulsion intime et influence extérieure. Voir par exemple «Le mauvais vitrier » à quoi j'ai emprunté la ci-devant distinction entre «satanique » et « hystérique » $(O C, I, 286)$ ou cette lettre au même Flaubert dans laquelle, pour expliquer « certaines actions
} 
remuent » $(O C, I, 5)$, et notre " grande hérésie » $(C P l, I, 337)$ devient « la plus belle de ses ruses » : celle « de nous persuader qu'il n'existe pas » (OC, $I, 327)$. « Sublime subtilité du diable » (182-3). Et je ne puis me défendre de la tentation de lire in absentia, dans les S de la faute, le chiffre du démon ${ }^{29}$. Prétendre à la sincérité faisait fourcher la langue : nier le diable, c'est encore l'appeler ${ }^{30}$. Et il serait même tentant de rêver qu'étant dans les détails, le diable se soit offert un dernier rire, quand, en 1861, la « langue » est remplacée par la « lettre ». Comme si Satan, pour se récompenser du rôle qu'il avait joué dans l'escamotage des $\mathrm{S}$, parvenait à se faire dédier le volume dans une dédicace que l'on voudrait dès lors pouvoir lire ainsi : au parfait magicien ès lettre S. Mais je vois des sourcils qui se haussent, laissons-là ces images, oublions-les s'il se peut et levons nos yeux de la faute pour, à l'instar de Baudelaire aveuglé, les reposer non loin de là.

Un peu au-dessous, d'abord, pour s'apercevoir que le souci de sincérité s'accompagna bien d'un souci d'atténuer les traces, sinon du péché originel, du moins du mal : dans le tour euphémique substituant au titre du recueil de belles «fleurs maladives $»{ }^{31}$. Mais c'est juste au-dessus de la faute qu'il faut surtout porter notre attention, sur l'expression qui, dans la dédicace, commute avec le «parfait magicien ». Car cette formule répétée ici à l'envi ne vint pas seule à l'esprit de Baudelaire pour faire l'éloge de son ami. La première ligne, écrite en caractères plus imposants encore, était en effet dédiée AU POËTE IMPECCABLE ${ }^{32}$. On a déjà noté la contradiction entre impeccabilité poétique et faute de langue. Mais il est temps de remarquer que l'interprétation que je viens de

ou pensées soudaines de l'homme » Baudelaire dit ne pas écarter « l'hypothèse de l'intervention d'une force méchante extérieure à lui » $(C P l, I I, 53)$. De son côté, Freud renversera la perspective en affirmant à plusieurs reprises que le recours au hasard ou à la superstition pour expliquer un acte manqué est une confirmation du rôle que l'inconscient a joué dans cet acte manqué (cf. par ex. Freud, 2001, 302).

${ }^{29}$ Je n'ai pas fait ma rhétorique chez Satan, le rusé doyen. Aussi, disons-le nettement : s'il était possible de l'assigner à une intention, la mise en rapport des $S$ disparus et d'un Satan prestidigitateur ne poserait, moyennant dispositio, aucun problème, car elle aurait pour elle non seulement l'intime conviction d'un lecteur, mais, fondant celle-ci, tout un faisceau concordant d'indices puisés dans l'œuvre. Ainsi, un lecteur aurait beau jeu d'assigner non au hasard mais au Poe de La lettre volée, le fait que le sceau subtilisé par le ministre, et celui qu'il lui substitue sont $\mathrm{D}$ et $\mathrm{S}$ et que le premier est presque explicitement mis pour « Devil ». Quant au second... (Voir aussi l'allitération suggestive de cette « sublime subtilité du diable » ou encore Henk Nuiten, p.298). On voudrait donc pouvoir maintenir la stabilité de l'impression en plongeant ce faisceau de «preuves » sous le niveau conscient de l'écriture et voir dans la « condensation » freudienne une sorte d'équivalent à la corrélation stylistique et ses différents avatars critiques.

30 « Item in eam, quæ fit cum expressa dæmonis invocatione, \& quæ fit cum tacita ejus invocatione, eo ipso quo adhibentur vana signa, tum enim dæmon immiscere se solet » (Dens 227). Il faut savoir varier ses autorités.

${ }^{31}$ Comparer cet euphémisme et le trope, exactement contraire du point de vue de l'intention ironique, qui consista, dans la dédicace du Spleen de Paris, à offrir en guise de recueil un « serpent » (Windels 2009).

${ }^{32}$ Ce tréma auquel nous sommes habitués n'est en fait publié qu'en 1861. En 1857, c'est l'accent grave qui avait prévalu, l'éditeur ne s'étant pas conformé au goût de Baudelaire. Mais on le trouve dans les brouillons et corrections d'épreuve (Dupont \& Pichois 969-70). 
suggérer se retrouve imprimée dans la matière même du texte : dans la formation de l'adjectif, dont le suffixe privatif et l'affaiblissement sémantique gomment le sens " peccable » sans en effacer les traces étymologique et sonore ${ }^{33}$. De même que le souci de sincérité n'évitait pas la scorie ironique du dédoublement de langue ; de même, et de façon plus nette, la négation du mal ne suffit décidément pas pour en arracher la racine, enfoncée dans le cœur de ce mot comme dans le cœur de l'homme. Et nous voici devant un nouveau soupçon d'intervention inconsciente : non plus faute de langue, mais choix d'un $\operatorname{mot}^{34}$.

Résumons-nous. La formule se renversait en lapsus sitôt que perçue ; le sens du mot en son contexte masque en les exhibant des connotations contraires ${ }^{35}$. Et ces deux couples articulés autour de la prise de conscience - formule/lapsus et sens/connotation distinguent entre ce qui fut visé et ce qui fut effectivement réalisé. Ainsi, le parfait souci de perfection, voire le sincère désir de sincérité constituaient d'évidents mobiles à la justification consciente de ces deux faits de langue : sens du mot ou formule. Mais dans le cas de l'erreur, ces mobiles conscients recouvraient deux couples de mobiles inconscients et contradictoires qui étaient cause du lapsus. Le premier m'intéressa peu : désir de sincérité et penchant à la vérité procédant au retour des réserves refoulées. L'autre, plus complexe, expliquait la perfection de cette imperfection langagière : le parfait souci de perfection trahissant un désir de légitimation, lui-même sapé par un désir simultané de "délégitimation". Mais j'ai tenté ensuite de suggérer que le lapsus produisait sur un autre plan un effet en contradiction littérale avec le sincère désir de sincérité: la double énonciation de l'ironie consciemment refusée étant comme illustrée par le pluriel inconsciemment oublié. Il y aurait donc un autre mobile inconscient à l'œuvre dans le lapsus et s'opposant au souci de sincérité, non plus le souci de vérité, mais quelque chose comme le souci plus grave ${ }^{36}$ de ne pas oublier la nature double du langage, ou l'impossibilité morale de faire l'économie de l'ironie. Ce ne serait donc pas la sincérité

\footnotetext{
${ }^{33}$ C'est ce que Freud appelle « figuration par le contraire », mais elle est parfaite : « la manière la plus facile de répliquer à une affirmation par un mot d'esprit consiste à soutenir son contraire et à confier à l'idée qui [vous] vient le soin d'éliminer, en donnant une interprétation autre, l'objection à l'égard de ce contraire qu'il y a lieu de redouter » (Freud, 1995, 312-3).

${ }_{34}$ « Je sais depuis longtemps qu'il est impossible de penser à un nombre ou à un nom dont le choix soit tout à fait arbitraire » (Freud, 2001, 302) ou: «Pour [qu'une] pensée devienne un mot d'esprit, il faut manifestement qu'un choix soit opéré parmi les formes d'expression possibles, afin que soit trouvée celle qui amènera le gain de plaisir procuré par les mots. L'auto-observation nous permet de savoir que ce n'est pas l'attention consciente qui fait ce choix » (Freud, 1995, 317). Il y a dans un de ces deux livres un passage qui se rapporte explicitement au choix stylistique d'un mot par un écrivain, mais je ne le retrouve pas.

${ }^{35}$ À nouveau, le paradigme de La Lettre volée où « ces mots-là, comme les enseignes et les affiches à lettres énormes, échappent à l'observateur par le fait même de leur excessive évidence » (Poe \& Baudelaire, 111).

${ }^{36}$ « Le lapsus a [...] neutralisé mon intention et révélé mon petit mensonge, en me faisant avancer une faute plus grave que celle dont j'étais réellement coupable » (Freud, 2001, 127).
} 
envers Gautier qui serait là remise en cause, mais la possibilité même de toute parole sincère, du moins en public ${ }^{37}$. On mesure la gradation, mais il reste à situer ces nouveaux mobiles dans l'économie générale du lapsus. Or, en ce qui concerne le choix du mot « impeccable », il est apparu un dernier couple de mobiles inconscients et contradictoires : le souci de ne pas oublier les traces en l'homme de la Chute, qui révèle par contraste une tendance à cet oubli, par ailleurs jugée coupable par Baudelaire ${ }^{38}$. Et je pose à présent que ces couples de mobiles ne sont que la reformulation d'une même opposition fondamentale entre espoir d'atteindre en public une parole sincère sans ironie, et vision du monde comme maudit. Opposition à la saveur toute baudelairienne, puisque une fois renversée, elle suppose que l'ironie a partie liée avec le souvenir de la Chute et qu'elle est « conscience dans le mal » $(O C, I, 80)$.

Voici donc le sens que j'assigne pour finir à l'erreur de Baudelaire. De même que l'homme est faillible parce qu'il est peccable, de même Baudelaire commet une faute de langue parce qu'il a commis, prétendant franchir sans ironie le "gouffre infranchissable [de] l'incommunicabilité » (696), une faute plus grave: non seulement «la grande hérésie », mais « le plus irrémédiable des vices » (323) : celui de s'illusionner sur son compte et sur celui de l'homme. Or, on ne peut gagner gratis le paradis de la parole : il faut payer sa dîme à l'ironie. Baudelaire n'est donc plus seulement trahi par le lapsus, il se rachète au contraire, par cette chute devant témoins, d'une trahison de soi plus grande : l'oubli, dans la recherche même de la formule, qu'une sincérité parfaite est, en public, impossible. Tout se passe donc comme si l'ironie du sort prenait le relais d'une ironie baudelairienne laissée à l'abandon, comme si le lapsus préservait dans l'inconscient même la conscience de la Chute, comme si Baudelaire enfin réussissait ce tour de force : s'être fidèle en se trompant.

\section{Devenirs d'une erreur et conclure}

\footnotetext{
${ }^{37}$ Sans invoquer la « sincérité » acquise dont parle Michel Butor, on pourrait aussitôt m'objecter les éclatants exemples de poèmes comme La servante au grand cœur... ou « L'Invitation au voyage » en vers. Mais dans le premier cas, il s'agit, pour citer un autre poème, de se rappeler « le vert paradis des amours enfantines » (OC, $I, 64$, je souligne) et dans le second, en effet, l'ironie s'éteint devant le seul lyrisme. Mais la parole lyrique est précisément pour Baudelaire le rêve d'une parole d'avant la Chute et le poète lyrique celui qui fait " retour vers [un] Éden perdu » (OC, II, 165). Bref, dans ces poèmes, nous sommes en poésie, tandis que dans la dédicace, nous y entrons. C'est même toute la spécificité du lieu dédicatoire : cela doit se faire en public. Et je ne peux, sur ce double problème que renvoyer ailleurs (Ross Chambers pour la « pratique de la dédicace baudelairienne », et Windels 2009, pour ces jardins de parole où la sincérité redevient possible).

38 «Il est certainement exact de décrire le refoulement comme le stade intermédiaire entre le réflexe de défense et le jugement de condamnation » (Freud, 1995, 314).
} 
Je voudrais, en guise d'épilogue, évoquer trois passages de l'œuvre postérieurs à la publication des Fleurs et dont on souhaiterait qu'en les écrivant Baudelaire, sans y renvoyer explicitement, songeât tout de même un petit peu à son parfait lapsus linguce. Voici le premier, tiré de Mon cœur mis à nu : « Je mettrai l'orthographe même sous la main du bourreau. (Th. Gautier)» (OC, I, 700 et note). La phrase n'a jusqu'à présent pas été retrouvée dans l'œuvre de Gautier, et il est probable que Baudelaire consigne ici le souvenir d'une conversation qui, peut-être, se sera tenue après la publication de la dédicace fautive. Mais l'on rêverait qu'elle ait couru sur ces langues qui avaient vu leur S tranché.

Nul besoin non plus de gloser la seconde citation, ni même d'y rêver bien longtemps : s'étonner suffira, se réjouir de la coïncidence et souligner que l'intention prêtée par Baudelaire au hasard vaut soupçon qu'il a parfois pu trouver quelque ironie à débrouiller dans les agissements du sort :

On se souvient d'avoir vu, à l'Exposition de 1855, d'excellents petits tableaux [...] signées du nom de Liès. Non loin d'eux, des tableaux exquis [...] portaient le nom de Leys. Presque le même peintre, presque le même nom. Cette lettre déplacée ressemble à un de ces jeux intelligents du hasard, qui a quelquefois l'esprit pointu comme un homme. (OC, II, 651)

Et le dernier passage, par quoi je terminerai : «Le jour où le jeune écrivain corrige sa première épreuve, il est fier comme un écolier qui vient de gagner sa première vérole » (OC, $I, 694)$. Ce mot d'esprit mériterait qu'on s'y attarde. Il ne fait pas seulement le lien implicite entre publication et prostitution ${ }^{39}$. Il $\mathrm{y}$ a, elliptiquement surgie entre la proposition principale et la relative inattendue, la prise de conscience a posteriori des conséquences d'un acte. Le caractère spirituel de cet aphorisme tient dans ce brusque dédoublement énonciatif où prend naissance l'ironie : l'écolier s'attendait benoîtement à perdre sa virginité, il nous est dit pour lui qu'il y gagna la vérole. Et ne dirait-on pas que Baudelaire se rendant compte, en 1857, qu'une faute lui a échappé en tête de recueil, ressemble un peu à cet écolier une fois qu'il se sera regardé dans quelque miroir ironique et aura reconnu sur son visage les premiers signes du mal français ? De même que le lapsus grêle la dédicace ${ }^{40}$. Entre le début et la fin de cette phrase, il y a la même distance qu'entre

\footnotetext{
${ }^{39}$ Précisément entre correction d'épreuves - voire correction des Fleurs - et défloration d'un collégien auprès d'une fille publique, si tant est que l'on puisse parler de « défloration » à propos d'un écolier mâle.

${ }^{40}$ Faut-il rappeler que Baudelaire avait ce poison dans ses veines et que celui-ci semblait avoir fini par matérialiser en lui le souvenir de la faute originelle ? (Pour rappel éventuel : Windels 2008). Analysant l'envoi d'un encrier à Mme Sabatier, qu'il rapporte à la contamination syphilitique, Michel Butor écrit de même : « La publicité de la maladie est comme une première esquisse de publication » (Butor 58).
} 
l'ignorance de la faute (qu'elle soit de langue ou originelle) et sa prise de conscience ironique.

Et si je ne peux pas affirmer que Baudelaire pensait effectivement aux Fleurs en écrivant ces trois bouts d'œuvre, je ne puis m'empêcher de penser que le dernier du moins est de la plume d'un homme qui avait su voir en lui ce quelque chose qui désirait si fort publier sa chute.

\section{Ouvrages cités}

Baudelaire, Charles. Euvres complètes. Vol. 1 \& 2. Paris : Gallimard, Pléiade, éd. Claude Pichois, 1976. Notés $O C, I$ et $O C, I I$.

—. Correspondance. Vol. 1 \& 2. Paris : Gallimard, Pléiade, éd. Claude Pichois, 1973. Notés $C P l, I$ et $C P l, I I$.

Butor, Michel. Histoire extraordinaire. Essai sur un rêve de Baudelaire. Paris : Gallimard, Folio essais, 1961.

Chambers, Ross : «Baudelaire et la pratique de la dédicace » Saggi et ricerche di letteratura francese, 1985 : 119-40.

Dens, Petrus. Theologia moralis et dogmatica. Dublin : Richard Coyne, 1832.

Freud, Sigmund. Psychopathologie de la vie quotidienne. Paris : Payot \& Rivages, 2001.

—. Le mot d'esprit et sa relation à l'inconscient. Paris : Gallimard, Folio essais, 1995.

—. Sur le rêve. Paris : Gallimard, Folio essais, 1991.

Grévisse, Maurice \& Goosse, André. Le bon usage. Paris - Louvain-la-Neuve : Duculot, 1993.

Liu, Bo. Les "Tableaux parisiens" de Baudelaire. Paris : L'Harmattan, 2003.

Nuiten, Henk. Les Variantes des «Fleurs du mal» et des «Épaves» de Charles Baudelaire (1821-1867). Étude de stylistique génétique. Amsterdam: Holland University Press, 1979.

Mensah, Alfred E. E. «Vers l'ontologie de la Dédicace des Fleurs du Mal » The French Review, Déc. 1997 : 237-50.

Pichois, Claude et Jacques, Dupont. L'Atelier de Baudelaire : "Les Fleurs du mal". Paris : Honoré Champion, 2005.

Poe, Edgar et Charles, Baudelaire. La Lettre volée. Histoires extraordinaires. Paris : Gallimard, Folio classiques, 2004. 
Pommier, Jean. Autour de l'édition originale des Fleurs du mal. Genève : Slatkine reprints, 1968.

Salvan, Jacques. «Le sens de la chute dans l'œuvre de Baudelaire » The French Review Déc. 1960 : 127-39.

Windels, Loïc. «Flaubert, Baudelaire, ou l'art de s'adresser aux femmes. » Fabula. Nov. $2008<$ www.fabula.org/colloques/document1075.php>.

—_ " Offrande publique et don privé : Baudelaire et le don du poème aux poètes », L'Offrande lyrique, éd. Jean-Nicolas Illouz, Paris : Hermann, 2009.

—_ « La dédicace à Maria Clemm : Baudelaire et le lapsus originel », à paraître en 2010. 